Bangladesh J. Zool. 48(2): 289-300, 2020

ISSN: 0304-9027

eISSN: $2408-8455$

\title{
DIVERSITY AND POPULATION DENSITY OF BIRDS AT THE JAHANGIRNAGAR UNIVERSITY CAMPUS, BANGLADESH
}

\author{
Delip K. Das*, Mohammad Mostafa Feeroz ${ }^{1}$ and Sajeda Begum ${ }^{1}$ \\ Department of Zoology, Jagannath University, Dhaka, Dhaka-1100, Bangladesh
}

\begin{abstract}
Rapid urbanization is changing the habitats of many wildlife species. Even so, certain species appear to adapt and now have thriving populations within cities. To look at the capacity for these spaces to provide "habitat" we examined population density of bird species using Jahangirnagar University campus close to the Dhaka mega city, Bangladesh. Four strip transects were surveyed from March 2008 to February 2009 (12 times/transect). A total of 101 species were found along the transect areas. The density of Lesser Whistling Duck (Dendrocygna javanica) was the highest 107.02indiv. / ha while Large-tailed Nightjar (Caprimulgus macrurus) was the lowest 0.01indiv. / ha among resident birds of Jahangirnagar University campus. Among the migratory birds, the density of Pintail Snipe (Gallinago stenura) and Common Stonechat (Saxicola torquata) were the highest (1.99indiv. / ha.) and the lowest (0.02indiv. / ha.), of the different available habitats, water bodies contained the highest density (318.59indiv. / ha) and grassland contained the lowest (2.48indiv./ha) of bird population. The percentage of average density of non-passerine and passerine birds was $65 \%$ and $35 \%$, respectively. Future studies of avian composition and density is recommended at a regular interval to understand changes in the carrying capacity and avian community of the campus as a function of altering habitat due urbanization.
\end{abstract}

Key words: Bangladesh, non-passerine, passerine, transect sampling, and urban

\section{INTRODUCTION}

Urbanization leads to habitat destruction, habitat loss and habitat fragmentation, as a result large continuous habitats are disconnected and divided into smaller unconnected patches. Habitat loss due to developmental activities like increase in roads and buildings exposes wildlife to new man-made stress. During the process of urbanization some animal species disappear or move other areas in search of food. Species that cannot move as fast as urbanization is expanding will disappear. Nonetheless, some species appear to adapt, increase in density and may have booming population within urban

*Author for corresponding: <bisharga1095@gmail.com>, 1Department of Zoology, Jahangirnagar university, Savar, Dhaka, Bangladesh.

(C2020 Zoological Society of Bangladesh DOI: https://doi.org/10.3329/bjz.v48i2.52369 
areas, such as crows, sparrows, doves, pigeons and so on. Thus, urbanization also filters bird communities. Natural habitat with planned plantations (like parks) within urban centers can be particularly attractive to many wildlife. This includes sometimes university campuses with diverse vegetation forming mosaic of various habitats including agricultural lands, orchards and botanical gardens in and around administrative building with human settlements. To look at the capacity for these spaces to provide "habitat" we examined population density of bird species using Jahangirnagar University campus close to the mega city of Dhaka, Bangladesh.

196 species of birds are recorded from Jahangirnagar University Campus that is almost 30\% of all the bird species that found in Bangladesh (Begum 2016). Much studies had been conducted on aspects of birds of Jahangirnagar University Campus. For instance, Feeroz et al. (1988), Begum (1997) and Mohsanin et al. (2009) studied species diversity and occurrence of birds. Begum (1992) and Hossain (1999) studied breeding ecology of birds. However, no study quantified the population density of birds in the campus. Therefore, this paper aimed to record bird diversity and investigate bird population density per hectare area in the campus.

\section{MATERIAL AND METHODS}

Study area: Jahangirnagar University, an area of about 280 hectares, is located at $23^{\circ} 52.764^{\prime} \mathrm{N}$ latitude and $90^{\circ} 16.068^{\prime} \mathrm{E}$ longitude (Fig. 1). The existing vegetation in this area is now of secondary character, originated from a tropical deciduous 'Sal' (Shorea robusta) forest community (Begum 2016). Most of the 'Sal' forest are lost as a result of urbanization. However, some isolated patches can still be seen along the Dhaka-Aricha highway. The land use is limited and protected by university campus authority, which allows preserving the remnant of the past. At present, the campus formed a mosaic of various habitats such as grasslands, woodlands, agricultural lands, water bodies (ponds and lakes), scrubland, stubbles, marsh and human settlement. Therefore, the university campus offers enormous feeding and roosting habitat, provides breeding facilities and makes a safe area for the birds. As a result, the campus is rich in avifaunal diversity.

Vegetation of the campus were classified into six categories. Human settlement consisted academic and administrative buildings, hostels, staff quarters and homestead gardens, mostly contained trees like Tectona grandis, Acacia auriculariformes, Swietenia mehagani, Casurina sp. Delonix regia, Artocarpus heterophyllus, Albizzia lebbeck, A. procera etc. Water body consisted mostly freshwater lakes and ponds. This habitat contained aquatic plants and dominated by water lilies during monsoon. Marshy area were low-lying areas found in 
northern, southern and central part of the campus. This habitat remained dry and are used as agricultural lands during winter whereas it filled with water and become marshy during monsoon. Grass land habitat was found in the southwestern portion of the campus, distributed variably behind the central library, beside the Bangabandhu Sheikh Mujib Hall, and inside the Wildlife Rescue Center. These areas were dominated by common sun grasses (Imperata cylindrica), mixed with diverse kinds of sedges and isolated patches of tall grasses. Woodland habitat was widely distributed in the campus. Large patch of Neem (Azadirachta indica) near "Gerua", of Jackfruit (Artocarpus heterophyllus) in eastern and central part of the campus, of various woody plants in botanical garden and Wildlife Rescue Centre consisted major woodland habitat in the campus. A. chaplasa, Acacia moniliformis, A. auricuriformis, Mangifera indica, Shorea robusta, etc. were common tree species. Bushy Area comprised of herbs, shrubs and scrub jungle were widely distributed in the campus around gardens and human settlements.

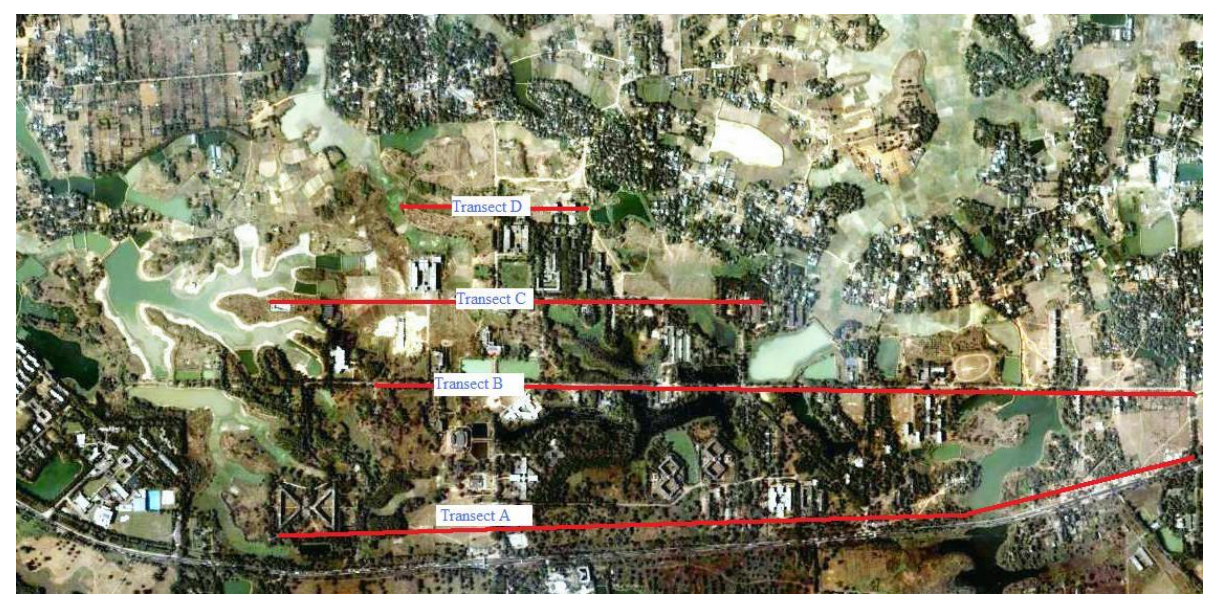

Fig. 1. Map of the study area showing position of four transects.

Data collection: The study was conducted from March 2008 to February 2009. Strip transect sampling method (Buckland et al. 2001) was found most suitable to estimate the population density. Four strip transects were selected and labeled as A, B, C and D. Start and end point's coordinates of all transects were marked by a Garmin GPS (Fig. 1). The length of the transects were 2.6, 2.3, 1.7 and $0.9 \mathrm{~km}$ for $\mathrm{A}, \mathrm{B}, \mathrm{C}$ and $\mathrm{D}$, respectively and the width of each transect was $20 \mathrm{~m}$. In every month, four days were spent surveying these transects. Survey were carried on foot at a speed of $1.5 \mathrm{~km} / \mathrm{h}$, with frequent stops for observing and counting birds. A total of 84 hours in 48 days were spent for data collection. The survey was 
conducted during active period of birds i.e. early morning ( $0630 \mathrm{~h}$ to $0830 \mathrm{~h}$ ) and late afternoon (1600h to $1800 \mathrm{~h})$.

Data Analysis: Data analysis was conducted using Excel 2007 platform. Population density was calculated by $D=n / A$, where $n=$ total counts of birds and $\mathrm{A}=$ area covered. Relative density was calculated in percentages.

\section{RESULTS AND DISCUSSION}

A total of 101 species were recorded within transects of which 80,17 and 4 were common, uncommon and rare respectively.(Fifty five) were non-passerine and 46 were passerine birds. Also, 23 species were categorized as migratory, 56 as breeding resident and 22 as non-breeding resident.

Population density: Population density varied between species, habitats, seasons and months. The mean density of Lesser Whistling Duck (Dendrocygna javanica) was the highest (107.02indiv./ha.) and Large-tailed Nightjar (Caprimulgus macrurus) was the lowest (0.01indiv./ha.) among resident birds. The average density of Pintail Snipe (Gallinago stenura) was the highest (1.99indiv./ha.) among migratory birds. Brown Shrike (Lanius cristatus) and Common Stonechat (Saxicola torquata) occurred in the lowest (0.02indiv. / ha.) density (Table 1). No previous study is available to compare the density of birds at JU but Mohsanin et. al. (2009) studied status and seasonal occurrence of birds in the campus where they reported Lesser Whistling Duck as very common species. This duck species is a winter visitor that congregates in huge number in the water bodies of the campus and therefore, it is ranked the top.

Habitat preference: Habitat of JU campus had been divided into grasslands and cultivated lands, woodland, marshy area, water bodies and human settlements. Water bodies contained the highest density (318.59indiv./ha) in December due to large flocks of Lesser Whistling Duck, the winter visitors. It contained the lowest in July (2.9indiv./ha) in absence of these waterfowls. In contrast, grasslands contained the lowest (2.48indiv./ha) in May and highest in November (16.47indiv./ha.). Human settlements, marshy areas, woodlands and bushy areas showed maximum density in November 33.85indiv./ha,47.13indiv. /ha,58.20/ha and 23.70indiv./ha, respectively while showed minimum density in July 15.97indiv./ha,6.25indiv./ha,25.69indiv./ha,8.30indiv. / ha, respectively.

Grassland density increased in November due to the migratory grassland species such as stonechats, shrikes, wagtails and pipits.

Relative density and Seasonal Variation: Relative density of different species varied between months in different habitats (Fig.2). Water bodies possessed $70 \%$ of the total bird population in March and $2.7 \%$ in September. Human settlements contained the highest $25.5 \%$ and the lowest $5.9 \%$ of the total 
Table 1. List of birds with Mean Density(/ha.) and Maximum-Minimum range at Jahangirnagar University

\begin{tabular}{|c|c|c|c|c|}
\hline S1. & Scientific Name & $\begin{array}{c}\text { English } \\
\text { name }\end{array}$ & $\begin{array}{c}\text { Mean Density } \\
\text { (/ha.) }\end{array}$ & $\begin{array}{c}\text { Range } \\
\text { (Max.-Min.) }\end{array}$ \\
\hline \multicolumn{5}{|c|}{$\begin{array}{l}\text { Order: Anseriformes } \\
\text { Family: Dendrocygnidae }\end{array}$} \\
\hline 1 & Dendrocygna bicolor & $\begin{array}{l}\text { Fulvous } \\
\text { Whistling- } \\
\text { duck }\end{array}$ & 0.19 & $0.28-0.14$ \\
\hline 2 & Dendrocygna javanica & $\begin{array}{l}\text { Lesser } \\
\text { Whistling- } \\
\text { duck }\end{array}$ & 107.02 & $312.42-0.5$ \\
\hline \multicolumn{5}{|c|}{ Family: Anatidae } \\
\hline 3 & Anus querquedula & Garganey & 0.33 & $0.43-0.28$ \\
\hline \multicolumn{5}{|c|}{$\begin{array}{l}\text { Order: Piciformes } \\
\text { Family: Picidae }\end{array}$} \\
\hline 4 & Dendrocopos macei & $\begin{array}{l}\text { Fulvous- } \\
\text { breasted } \\
\text { Woodpecker }\end{array}$ & 0.38 & $3.76-0.07$ \\
\hline 5 & Dinopium benghalense & $\begin{array}{l}\text { Black- } \\
\text { rumped } \\
\text { Flameback }\end{array}$ & 0.37 & $0.71-0.14$ \\
\hline 6 & Jynx torquilla & $\begin{array}{l}\text { Eurasian } \\
\text { Wryneck }\end{array}$ & 0.19 & $0.28-0.14$ \\
\hline 7 & Micropternus brachyrus & $\begin{array}{l}\text { Rufous } \\
\text { Woodpecker }\end{array}$ & 0.07 & $0.07-0.07$ \\
\hline \multicolumn{5}{|c|}{ Family: Megalaimidae } \\
\hline 8 & Megalaima haemacephala & $\begin{array}{l}\text { Coppersmith } \\
\text { Barbet }\end{array}$ & 0.66 & $2.77-0.07$ \\
\hline \multicolumn{5}{|c|}{$\begin{array}{l}\text { Order: Upupiformes } \\
\text { Family: Upupidae }\end{array}$} \\
\hline 9 & Upupa epops & $\begin{array}{l}\text { Common } \\
\text { Hoopoe }\end{array}$ & 0.07 & $0.07-0.07$ \\
\hline \multicolumn{5}{|c|}{$\begin{array}{l}\text { Order: Coraciformes } \\
\text { Family: Coraciidae }\end{array}$} \\
\hline 10 & Coracias benghalensis & Indian Roller & 0.07 & $0.07-0.07$ \\
\hline \multicolumn{5}{|c|}{ Family: Alcedinidae } \\
\hline 11 & Alcedo atthis & $\begin{array}{l}\text { Common } \\
\text { Kingfisher }\end{array}$ & 0.37 & $0.64-0.14$ \\
\hline \multicolumn{5}{|c|}{ Family: Halcyonidae } \\
\hline 12 & Halcyon smyrnensis & $\begin{array}{l}\text { White- } \\
\text { throated } \\
\text { Kingfisher }\end{array}$ & 0.38 & $0.71-0.21$ \\
\hline 13 & Halcyon capensis & $\begin{array}{l}\text { Stork-billed } \\
\text { Kingfisher }\end{array}$ & 0.24 & $0.5-0.07$ \\
\hline \multicolumn{5}{|c|}{ Family: Cerylidae } \\
\hline 14 & Ceryle rudis & $\begin{array}{l}\text { Pied } \\
\text { Kingfisher }\end{array}$ & 0.35 & $0.35-0.35$ \\
\hline \multicolumn{5}{|c|}{ Family: Meropidae } \\
\hline & Merops orientalis & $\begin{array}{l}\text { Green Bee- } \\
\text { eater }\end{array}$ & 0.67 & $0.92-0.43$ \\
\hline \multicolumn{5}{|c|}{$\begin{array}{l}\text { Order: Cuculiformes } \\
\text { Family: Cuculidae }\end{array}$} \\
\hline 16 & Cacomantis merulinus & $\begin{array}{l}\text { Plaintive } \\
\text { Cuckoo }\end{array}$ & 0.14 & $0.14-0.14$ \\
\hline 17 & Clamator jacobinus & Jacobin & 0.07 & $0.07-0.07$ \\
\hline
\end{tabular}




\begin{tabular}{|c|c|c|c|c|}
\hline S1. & Scientific Name & $\begin{array}{c}\text { English } \\
\text { name }\end{array}$ & $\begin{array}{c}\text { Mean Density } \\
\text { (/ha.) }\end{array}$ & $\begin{array}{c}\text { Range } \\
\text { (Max.-Min.) }\end{array}$ \\
\hline & & Cuckoo & & \\
\hline 18 & Cuculus micropterus & $\begin{array}{l}\text { Indian } \\
\text { Cuckoo }\end{array}$ & 0.18 & $0.28-0.07$ \\
\hline 19 & Eudynamys scolopacea & Asian Koel & 1.31 & $2.34-0.35$ \\
\hline 20 & Hierococcyx varius & $\begin{array}{l}\text { Common } \\
\text { Hawk } \\
\text { Cuckoo }\end{array}$ & 0.75 & $1.85-0.07$ \\
\hline \multicolumn{5}{|c|}{ Family: Centropodidae } \\
\hline 21 & Centropus sinensis & $\begin{array}{l}\text { Greater } \\
\text { Coucal }\end{array}$ & 0.14 & $0.21-0.07$ \\
\hline \multicolumn{5}{|c|}{$\begin{array}{l}\text { Order: Psittaciformes } \\
\text { Family: Psittacidae }\end{array}$} \\
\hline 22 & Psittacula krameri & $\begin{array}{l}\text { Rose-ringed } \\
\text { Parakeet }\end{array}$ & 0.48 & $1.28-0.14$ \\
\hline \multicolumn{5}{|c|}{$\begin{array}{l}\text { Order: Apodiformes } \\
\text { Family: Apodidae }\end{array}$} \\
\hline 23 & Apus nipalensis & House Swift & 1.25 & $3.41-0.14$ \\
\hline 24 & Cypsiurus balasiensis & $\begin{array}{l}\text { Asian Palm } \\
\text { Swift }\end{array}$ & 0.76 & $1.35-0.35$ \\
\hline \multicolumn{5}{|c|}{$\begin{array}{l}\text { Order: Strigiformes } \\
\text { Family: Tytonidae }\end{array}$} \\
\hline \multicolumn{5}{|c|}{ Family: Strigidae } \\
\hline 26 & Athene brama & $\begin{array}{l}\text { Spotted } \\
\text { Owlet }\end{array}$ & 0.21 & $0.21-0.21$ \\
\hline \multicolumn{5}{|c|}{ Family: Caprimulgidae } \\
\hline 27 & Caprimulgus macrurus & $\begin{array}{l}\text { Large-tailed } \\
\text { Nightjar }\end{array}$ & 0.01 & $0.07-0.01$ \\
\hline \multicolumn{5}{|c|}{$\begin{array}{l}\text { Order: Columbiformes } \\
\text { Family: Columbidae }\end{array}$} \\
\hline 28 & Columba livia & Rock Pigeon & 0.90 & $1.42-0.43$ \\
\hline 29 & Streptopelia chinensis & Spotted Dove & 3.16 & $4.47-1.14$ \\
\hline 30 & Streptopelia tranquebarica & $\begin{array}{l}\text { Red-collard } \\
\text { Dove }\end{array}$ & 0.57 & $0.71-0.5$ \\
\hline 31 & Treron phoenicoptera & $\begin{array}{l}\text { Yellow-footed } \\
\text { Green Pigeon }\end{array}$ & 6.17 & $11.07-1.28$ \\
\hline \multicolumn{5}{|c|}{$\begin{array}{l}\text { Order: Gruiformes } \\
\text { Family: Rallidae }\end{array}$} \\
\hline 32 & Amaurornis phoenicurus & $\begin{array}{l}\text { White- } \\
\text { breasted } \\
\text { Waterhen }\end{array}$ & 0.42 & $0.99-0.07$ \\
\hline 33 & Gallinula chloropus & $\begin{array}{l}\text { Common } \\
\text { Moorhen }\end{array}$ & 0.62 & $0.99-0.28$ \\
\hline \multicolumn{5}{|c|}{$\begin{array}{l}\text { Order: Ciconiformes } \\
\text { Family: Scolopacidae }\end{array}$} \\
\hline 34 & Actitis hypoleucos & $\begin{array}{l}\text { Common } \\
\text { Sandpiper }\end{array}$ & 0.28 & $0.57-0.14$ \\
\hline 35 & Gallinago stenura & $\begin{array}{l}\text { Pin-tailed } \\
\text { Snipe }\end{array}$ & 1.99 & $6.1-0.07$ \\
\hline 36 & Tringa ochropus & $\begin{array}{l}\text { Green } \\
\text { Sandpiper }\end{array}$ & 0.40 & $0.17-0.07$ \\
\hline 37 & Tringa glareola & $\begin{array}{l}\text { Wood } \\
\text { Sandpiper }\end{array}$ & 0.31 & $0.43-0.21$ \\
\hline
\end{tabular}




\begin{tabular}{|c|c|c|c|c|}
\hline S1. & Scientific Name & $\begin{array}{c}\text { English } \\
\text { name }\end{array}$ & $\begin{array}{c}\text { Mean Density } \\
\text { (/ha.) }\end{array}$ & $\begin{array}{c}\text { Range } \\
\text { (Max.-Min.) }\end{array}$ \\
\hline 38 & Rostratula benghalensis & $\begin{array}{l}\text { Painted } \\
\text { Snipe }\end{array}$ & 0.83 & $1.28-0.21$ \\
\hline \multicolumn{5}{|c|}{ Family: Jacanidae } \\
\hline 39 & Metopidius indicus & $\begin{array}{l}\text { Bronze- } \\
\text { winged } \\
\text { Jacana }\end{array}$ & 0.42 & $0.78-0.14$ \\
\hline \multicolumn{5}{|c|}{ Family: Charadridae } \\
\hline 40 & Vanellus cinereus & $\begin{array}{l}\text { Grey-headed } \\
\text { Lapwing }\end{array}$ & 1.74 & $2.2-1.28$ \\
\hline 41 & Vanellus indicus & $\begin{array}{l}\text { Red-wattled } \\
\text { Lapwing }\end{array}$ & 0.20 & $0.43-0.07$ \\
\hline 42 & Vanellus malabaricus & $\begin{array}{l}\text { Yellow- } \\
\text { wattled } \\
\text { Lapwing }\end{array}$ & 0.23 & $0.28-0.07$ \\
\hline \multicolumn{5}{|c|}{ Family: Accipitridae } \\
\hline 43 & Elanus caeruleus & $\begin{array}{l}\text { Black- } \\
\text { shouldered } \\
\text { Kite }\end{array}$ & 0.09 & $0.14-0.07$ \\
\hline 44 & Haliastur Indus & $\begin{array}{l}\text { Brahminy } \\
\text { Kite }\end{array}$ & 0.26 & $0.35-0.14$ \\
\hline 45 & Milvus migrans & Black Kite & 0.20 & $0.43-0.07$ \\
\hline 46 & Spilornis cheela & $\begin{array}{l}\text { Crested } \\
\text { Serpent } \\
\text { Eagle }\end{array}$ & 0.07 & \\
\hline \multicolumn{5}{|c|}{ Family: Phalacrocoracidae } \\
\hline 47 & Phalacrocorax niger & $\begin{array}{l}\text { Little } \\
\text { Cormorant }\end{array}$ & 0.16 & $0.35-0.07$ \\
\hline \multicolumn{5}{|c|}{ Family: Ardeidae } \\
\hline 48 & Ardeola grayii & $\begin{array}{l}\text { Indian Pond } \\
\text { Heron }\end{array}$ & 1.55 & $2.13-0.92$ \\
\hline 49 & Bubulcus ibis & Cattle Egret & 0.55 & $1.85-0.21$ \\
\hline 50 & Butorides striatus & Little Heron & 0.13 & $0.21-0.07$ \\
\hline 51 & Casmerodius albus & Great Egret & 0.09 & $0.21-0.07$ \\
\hline 52 & Egretta garzetta & Little Egret & 0.59 & $1.63-0.07$ \\
\hline 53 & Ixobrychus cinnamomeus & $\begin{array}{l}\text { Cinnamon } \\
\text { Bittern }\end{array}$ & 0.09 & $0.14-0.07$ \\
\hline 54 & Mesophoyx intermedia & $\begin{array}{l}\text { Intermediate } \\
\text { Egret }\end{array}$ & 0.84 & $5.04-0.07$ \\
\hline \multicolumn{5}{|c|}{ Family: Ciconiidae } \\
\hline 55 & Anastomus oscitans & $\begin{array}{l}\text { Asian } \\
\text { Openbill }\end{array}$ & 3.87 & $7.34-1.42$ \\
\hline \multicolumn{5}{|c|}{$\begin{array}{l}\text { Order: Passeriformes } \\
\text { Family: Laniidae }\end{array}$} \\
\hline 56 & Lanius cristatus & $\begin{array}{l}\text { Brown } \\
\text { Shrike }\end{array}$ & 0.02 & $0.02-0.02$ \\
\hline 57 & Lanius schach & $\begin{array}{l}\text { Long-tailed } \\
\text { Shrike }\end{array}$ & 0.75 & $1.42-0.28$ \\
\hline \multicolumn{5}{|c|}{ Family: Corvidae } \\
\hline 58 & Aegithina tiphia & $\begin{array}{l}\text { Common } \\
\text { Iora }\end{array}$ & 0.43 & $1.42-0.28$ \\
\hline 59 & Artamus fuscus & $\begin{array}{l}\text { Ashy Woods } \\
\text { wallow }\end{array}$ & 1.10 & $2.7-0.21$ \\
\hline 60 & Coracina macei & Large & 0.07 & $0.07-0.07$ \\
\hline
\end{tabular}




\begin{tabular}{|c|c|c|c|c|}
\hline S1. & Scientific Name & $\begin{array}{c}\text { English } \\
\text { name }\end{array}$ & $\begin{array}{c}\text { Mean Density } \\
\text { (/ha.) }\end{array}$ & $\begin{array}{c}\text { Range } \\
\text { (Max.-Min.) }\end{array}$ \\
\hline & & $\begin{array}{l}\text { Cuckoo- } \\
\text { shrike }\end{array}$ & & \\
\hline 61 & Coracina melanoptera & $\begin{array}{l}\text { Black- } \\
\text { headed } \\
\text { Cuckoo- } \\
\text { shrike }\end{array}$ & 0.37 & $0.57-0.07$ \\
\hline 62 & Corvus macrorhynchos & $\begin{array}{l}\text { Large-billed } \\
\text { Crow }\end{array}$ & 0.92 & $1.7-0.28$ \\
\hline 63 & Corvussplendens & House Crow & 4.62 & $8.73-1.49$ \\
\hline 64 & Dendrocitta vagabunda & $\begin{array}{l}\text { Rufous } \\
\text { Treepie }\end{array}$ & 0.57 & $1.06-0.28$ \\
\hline 65 & Dicrurus macrocercus & $\begin{array}{l}\text { Black } \\
\text { Drongo }\end{array}$ & 3.57 & $5.54-2.13$ \\
\hline 66 & Oriolus oriolus & $\begin{array}{l}\text { Eurasian } \\
\text { Golden } \\
\text { Oriole }\end{array}$ & 0.07 & $0.07-0.07$ \\
\hline 67 & Oriolus xanthornus & $\begin{array}{l}\text { Black- } \\
\text { hooded } \\
\text { Oriole }\end{array}$ & 1.14 & $1.7-0.99$ \\
\hline 68 & Pericrocotus cinnamomeus & $\begin{array}{l}\text { Small } \\
\text { Minivet }\end{array}$ & 0.43 & $0.57-0.14$ \\
\hline 69 & Tephrodornis gularis & $\begin{array}{l}\text { Large } \\
\text { Woodshrike }\end{array}$ & 0.07 & $0.07-0.07$ \\
\hline 70 & Tephrodornis pondicerianus & $\begin{array}{l}\text { Common } \\
\text { Woodshrike }\end{array}$ & 0.14 & $0.14-0.14$ \\
\hline \multicolumn{5}{|c|}{ Family: Muscicapidae } \\
\hline 71 & Copsychus saularis & $\begin{array}{l}\text { Oriental } \\
\text { Magpie } \\
\text { Robin }\end{array}$ & 1.54 & $2.63-0.85$ \\
\hline 72 & Ficedula parva & $\begin{array}{l}\text { Red-throated } \\
\text { Flycatcher }\end{array}$ & 0.18 & $0.28-0.07$ \\
\hline 73 & Saxicola torquata & $\begin{array}{l}\text { Common } \\
\text { Stonechat }\end{array}$ & 0.02 & $0.02-0.02$ \\
\hline 74 & Zoothera citrine & $\begin{array}{l}\text { Orange- } \\
\text { headed } \\
\text { Thrush }\end{array}$ & 0.20 & $0.5-0.07$ \\
\hline \multicolumn{5}{|c|}{ Family: Sturnidae } \\
\hline 75 & Acridotheres fuscus & Jungle Myna & 3.04 & $5.82-0.92$ \\
\hline 76 & Acridotheres ginginianus & Bank Myna & 0.21 & $0.21-0.21$ \\
\hline 77 & Acridothere tristis & $\begin{array}{l}\text { Common } \\
\text { Myna }\end{array}$ & 9.92 & $19.45-7.24$ \\
\hline 78 & Sturnus Contra & $\begin{array}{l}\text { Asian Pied } \\
\text { Starling }\end{array}$ & 8.40 & $15.61-3.48$ \\
\hline 79 & Sturnus malabaricus & $\begin{array}{l}\text { Chestnut- } \\
\text { tailed } \\
\text { Starling }\end{array}$ & 1.39 & $3.69-0.21$ \\
\hline \multicolumn{5}{|c|}{ Family: Certhidae } \\
\hline 80 & Parus major & Great Tit & 0.23 & $0.57-0.07$ \\
\hline \multicolumn{5}{|c|}{ Family: Hirundinidae } \\
\hline 81 & Cecropis daurica & $\begin{array}{l}\text { Red-rumped } \\
\text { Swallow }\end{array}$ & 0.05 & $0.43-0.14$ \\
\hline 82 & Hirundo rustica & $\begin{array}{l}\text { Barn } \\
\text { Swallow }\end{array}$ & 1.42 & $1.49-1.28$ \\
\hline
\end{tabular}




\begin{tabular}{|c|c|c|c|c|}
\hline S1. & Scientific Name & $\begin{array}{c}\text { English } \\
\text { name }\end{array}$ & $\begin{array}{c}\text { Mean Density } \\
\text { (/ha.) }\end{array}$ & $\begin{array}{c}\text { Range } \\
\text { (Max.-Min.) }\end{array}$ \\
\hline \multicolumn{5}{|c|}{ Family: Pycnonotidae } \\
\hline 83 & Pycnonotus cafer & $\begin{array}{l}\text { Red-vented } \\
\text { Bulbul }\end{array}$ & 4.42 & $7.52-2.91$ \\
\hline \multicolumn{5}{|c|}{ Family: Cisticolidae } \\
\hline 84 & Cisticola juncidis & $\begin{array}{l}\text { Zitting } \\
\text { Cisticola }\end{array}$ & 0.66 & $1.92-0.14$ \\
\hline \multicolumn{5}{|c|}{ Family: Zosteropidae } \\
\hline 85 & Zosterops palpebrosus & $\begin{array}{l}\text { Oriental } \\
\text { White-eye }\end{array}$ & 0.30 & $0.71-0.14$ \\
\hline \multicolumn{5}{|c|}{ Family: Sylviidae } \\
\hline 86 & Orthotomus sutorius & $\begin{array}{l}\text { Common } \\
\text { Tailorbird }\end{array}$ & 1.22 & $2.27-0.43$ \\
\hline 87 & Phylloscopus collybita & $\begin{array}{l}\text { Common } \\
\text { Chiffchaff }\end{array}$ & 0.09 & $0.14-0.07$ \\
\hline 88 & Phylloscopus fuscatus & $\begin{array}{l}\text { Dusky } \\
\text { Warbler }\end{array}$ & 0.11 & $0.14-0.07$ \\
\hline 89 & Turdoides earlei & $\begin{array}{l}\text { Striated } \\
\text { Babbler }\end{array}$ & 0.41 & $0.78-0.07$ \\
\hline 90 & Turdoides striatus & $\begin{array}{l}\text { Jugnle } \\
\text { Babbler }\end{array}$ & 4.53 & $66.6-2.48$ \\
\hline \multicolumn{5}{|c|}{ Family: Alaudidae } \\
\hline 91 & Mirafra assamica & $\begin{array}{l}\text { Rufous- } \\
\text { winged } \\
\text { Bushlark }\end{array}$ & 0.28 & $0.57-0.07$ \\
\hline \multicolumn{5}{|c|}{ Family: Nectariniidae } \\
\hline 92 & Dicaeum erythrorynchos & $\begin{array}{l}\text { Pale-billed } \\
\text { Flowerpecker }\end{array}$ & 0.09 & $0.14-0.07$ \\
\hline 93 & Cinnyris asiaticus & $\begin{array}{l}\text { Purple } \\
\text { Sunbird }\end{array}$ & 0.12 & $0.28-0.07$ \\
\hline 94 & Leptocoma zeylonica & $\begin{array}{l}\text { Purple- } \\
\text { rumped } \\
\text { Sunbird }\end{array}$ & 0.33 & $0.85-0.14$ \\
\hline \multicolumn{5}{|c|}{ Family: Passeridae } \\
\hline 95 & Anthus hodgsoni & $\begin{array}{l}\text { Olive-backed } \\
\text { Pipit }\end{array}$ & 0.13 & $0.14-0.07$ \\
\hline 96 & Anthus rufulus & $\begin{array}{l}\text { Paddyfield } \\
\text { Pipit }\end{array}$ & 0.33 & $0.99-0.07$ \\
\hline 97 & Lonchura malacca & $\begin{array}{l}\text { Black- } \\
\text { headed } \\
\text { Munia }\end{array}$ & 0.21 & $0.21-0.21$ \\
\hline 98 & Lonchura punctulata & $\begin{array}{l}\text { Scaly- } \\
\text { breasted } \\
\text { Munia }\end{array}$ & 2.37 & $8.16-0.14$ \\
\hline 99 & Motacilla alba & $\begin{array}{l}\text { White } \\
\text { Wagtail }\end{array}$ & 0.57 & $1.56-0.07$ \\
\hline 100 & Motacilla maderaspatensis & $\begin{array}{l}\text { White- } \\
\text { browed } \\
\text { Wagtail }\end{array}$ & 0.11 & $0.21-0.07$ \\
\hline 101 & Passer domesticus & $\begin{array}{l}\text { House } \\
\text { Sparrow }\end{array}$ & 3.94 & $9.72-1.56$ \\
\hline
\end{tabular}

population in July and March, respectively. Marshy areas showed the highest $25.5 \%$ and the lowest $3.6 \%$ of the total bird population in November and March, 
respectively. In grassland $8.6 \%$ and $0.7 \%$ of the total bird population was found in November and February. Woodlands and bushy areas possessed the highest $42.9 \%$ and $12.9 \%$ of the total bird population in August and September, respectively (Fig. 2).The highest population density was found during winter (947.66indiv./ha) and the lowest was found during post monsoon (184.39/ha). Summer and monsoon showed the density of 483indiv./ha. and 265indiv./ha. respectively.

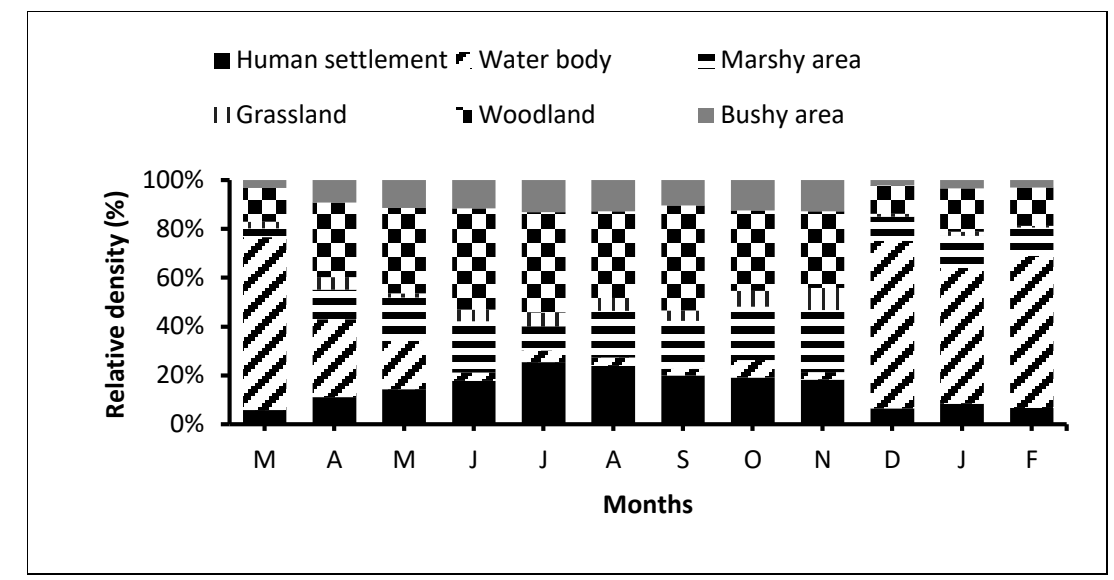

Fig. 2. Monthly relative density of bird's population of different habitats at JU campus.

Winter visitors and migrants are reason for increased density during the winter season in the campus and absence of them is the cause of lowest density in post monsoon. Presence of summer visitor such as cuckoos explain the increased density during summer and monsoon.

Passerine and Non-passerine bird: Non-passerines had the highest density (332.43indiv. /ha) in December but number of species of passerines were more $(n=29$.) than that of non-passerines $(n=27)$. Their lowest density (8.59indiv./ha) was found in July. The highest (82.9indiv./ha) and lowest (33.07indiv./ha) density of passerine birds was found in November and July respectively (Fig.3). The percentage of average density of passerine and non-passerine birds was $35 \%$ and $65 \%$, respectively during the study period. The average density of Common Myna was found the highest (9.92indiv./ha.) while Large Wood Shrike (Tephrodornis gularis) and Eurasian Golden Oriole (Oriolus oriolus) was found to be the lowest (0.01indiv./ha) in passerine birds. Lesser Whistling Duck (80.25indiv./ha.) was ranked the highest while Rufous Woodpecker and Largetailed Nightjar ranked the lowest (0.01indiv./ha.) in mean density among the non-passerine birds. 


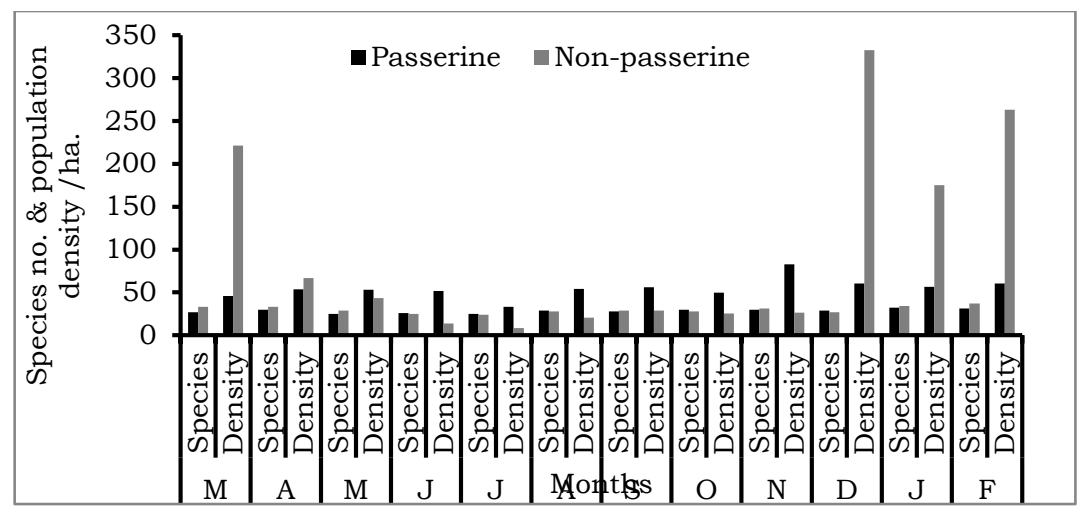

Fig. 3. Monthly species and population density variation of Passerine (dark bar) and Non-passerine (light bar) birds at JU campus.

Ducks, shorebirds and storks belongs to non-passerine group that compose large flock, disperse and migrate to short to long distances. Due to the high number of these species during winter increased the density. Even though there are migrant passerine such as warblers, pipits and wagtails but they don't form a large flock like non-passerines and explains the lower density of passerines in the campus. Common Myna is habituated with the human habitation and common in urban to semi-urban environment and therefore, was common in the campus. The habitat for Large-tailed Nightjar is not available everywhere in the campus in addition to their high camouflaging to diurnal habitat. There is possibility of underestimate of nightjar and owl for this reason. In conclusion, this study found 101 species of birds, of which only 13 species have density greater than 3indiv./ha. Future studies of avian composition and their density is recommended at a regular interval to understand the changes in the avian community as a function of altering habitat due urbanization in the campus.

Acknowledgement: We thank Jennifer Lewis for commenting and improving the first draft.

\section{LITERATURE CITED}

BEGUM, S. 2016. Birds of Jahangirnagar University Campus. Arannayk Foundation Bangladesh. 96pp.

BEGUM, S. 1997. Avifauna of Jahangirnagar University Campus, MSc thesis, Department of Zoology, Jahangirnagar University, Dhaka.

BEGUM, S. 1992. Breeding activities of nine bird species at Jahangirnagar University Campus, MSc thesis, Department of Zoology, Jahangirnagar University, Dhaka. 
BUCKLAND, S.T., ANDERSON, D.R., BURNHAM, K.P., LAAKE, J.L., BORCHERS, D.L. and THOMAS, L.2001. Introduction to distance sampling: estimating abundance of biological populations. Oxford University Press, Oxford.432pp.

FEEROZ, M.M., ZAVED, A. Al and ISLAM, M.A. 1988. A checklist of medicinal plants, freshwater organisms and vertebrate fauna of the Jahangirnagar University Campus. Bangladesh J.Life sci. 1(1):65-85.

GRIMMETT, R., INSKIPP, C. and INSKIPP, T. 1999. Pocket Guide to the Birds of the Indian Subcontinent. Oxford University Press, New Delhi. 384 pp.

HOSSAIN, M. 1999. Study on resident, migrant and breeding birds of Jahangirnagar University Campus, MSc thesis, Department of Zoology, Jahangirnagar University, Dhaka.

KHAN, M.M.H 2008. Protected Areas of Bangladesh - A Guide to Wildlife, Nishorgo Program, Bangladesh Forest Department, Dhaka, Bangladesh.

KREBS, C.J. 1999. Ecological Methodology, 2nd ed. Benjamin/ cummings, Menlow Park, CA.

MOHSANIN, S. and KHAN, M.M.H. 2009. Status and seasonal occurrence of the birds in Jahangirnagar University Campus, Bangladesh. Bangladesh J. Life Sci. 21 (1): 29-37.

OGUTU, J.O.,BHOLA,N., PIEPHO, H.P and REID, R. 2006. Efficiency of strip and line- transect surveys of African savannah mammals. J. Zool. 269: 149-160.

PRIMACK, R.B. 1993. Introduction to Conservation Biology. Sinauer Associates, Sunderland, MA.

SUTHERLAND, W.J. 1996. Ecological Census Techniques. A Handbook. Cambridge University Press, Cambridge.

(Manuscript received on 14 March, 2020 revised on 5 September 2020) 